\title{
Cognitive reserve: the evolution of the conceptual framework
}

\author{
Laura Serra $^{* *}$, Carlo Caltagirone ${ }^{2,3}$, and Marco Bozzali ${ }^{1}$ \\ ${ }^{1}$ Neuroimaging Laboratory, Santa Lucia Foundation, IRCCS, Via Ardeatina, 306, 00179, Rome, Italy \\ ${ }^{2}$ Department of Clinical and Behavioural Neurology, Santa Lucia Foundation, IRCCS, Via Ardeatina, 306, 00179, Rome, Italy \\ ${ }^{3}$ Department of Systems Medicine, University of Rome 'Tor Vergata', Via Montpellier, 100133, Rome, Italy
}

\begin{abstract}
Brain and Cognitive reserve concept is a theoretical general framework introduced to explain individual differences to withstand brain damages. Several behavioural and neuroimaging studies support the evidence that lifestyles act on brain plasticity modulating the impact of neurological insults. However, once that AD is clinically evident, the protective benefits of premorbid experience are assumed to be substantially decreased. This review presents an overview of the principal literature focused on reserve mechanisms. The evolution of the conceptual framework is considered, passing from brain and cognitive to neural reserve's hypothesis. Interactions between reserve mechanisms and $\mathrm{AD}$-related biomarkers are reported. In particular this review is focussed on the neuroimaging (MRI) studies. MRI provides the opportunity to investigate in vivo structural and functional brain changes. Several studies reported here assessed the impact of reserves on brain resilience. Literature supports the hypothesis that reserves impact on neurodegenerative process in the early phase of AD. Moreover, studies fit with the existence of a "neural reserve", characterised by specific neural networks and their efficiency. It remains to be demonstrated whether interventions later in life can modulate this brain resilience.
\end{abstract}

\section{Introduction}

Alzheimer's disease $(\mathrm{AD})$ is the most common cause of cognitive decline in west world's population [1]. It is a neurodegenerative disorder that typically presents with an isolated memory deficit, followed by a progressive accumulation of cognitive disabilities until conversion to dementia [1]. However, it is becoming increasingly clearer that there is a non-linear relationship between the extent of brain tissue damage and the resulting patients' clinical outcome [2,3].

To account for these apparent inconsistencies between brain tissue damage and clinical manifestations, it has been introduced the concept of "reserve" [2,3]. The concept of a reserve's capacity of the brain appeared for the first time in 1940, when unknown Authors stated that reserve's tissue was highly available in many organs of body including the brain [4]. According with this view, pathological lesions in the brain resulted in an extensive destruction of nervous tissue with a little derangement of function [4]. In the last thirty years reserve's concept has experienced several modifications.

In the traditional framework two different mechanisms were postulated for reserves: the brain reserve (BR) and the cognitive reserve (CR). BR referred to differences in brain size and other quantitative aspects of the brain (e.g. amount of neurons or synapses) that explain differential susceptibility to functional impairment in presence of pathology or neurological insult [5]. This concept arose by the observation that prevalence of dementia is lower in individuals with larger brains [6-8]. The BR implied a passive model of reserve, suggesting that the brain tolerates more accumulation of neuropathology before it reaches a critical threshold for the appearance of clinical symptoms [5]. Nevertheless, the CR implied the differences in cognitive processes as a function of lifetime intellectual activities and other environmental factors that explain the non-linear relationship between the severity of patients' brain damage and the correspondent clinical symptoms. The CR suggested that the brain actively copes with brain damage by using the pre-existing cognitive processes or by enlisting compensatory mechanisms $[3,9]$. The CR model hypothesised that cognitive processes are crucial for explaining individual' differences despite equal brain changes or pathology [10]. Cognitive processes consist of differences in cognitive efficiency, capacity or flexibility resulting from life experiences.

Stern and co-workers [11] addressed the concept of CR in a study on $\mathrm{AD}$ incidence. Analysing data from a large cohort of non-demented individuals, the Authors assumed that $\mathrm{AD}$ pathology slowly develops over time independently of $\mathrm{CR}$, and that the pathology begins many years before the onset of clinically diagnosed AD. Individuals with greater reserves should be able to tolerate $\mathrm{AD}$ pathology better than those with lower CR levels. As a consequence, $\mathrm{AD}$ onset should be delayed in subjects with high reserve. However, in subjects with high CR cognitive decline is faster after the inflection point (Figure 1). A neuropathological study showed that CR is more able than BR to withstand neurodegeneration [8]. Indeed, individuals with high CR (as measured by education) and low BR (as measured by head size) developed dementia less than subjects with low CR and low BR [8]. During the last years, emerged that the CR relied on current neural activity to explain functional differences, suggesting that neuronal

Correspondence to: Dr. Laura Serra, Via Ardeatina 306, 00179 Rome, Italy. Tel: 39-06-5150 1547; Fax: 39-06-5150 1213; Email address: 1.serra@hsantalucia.it

Key words: brain reserve, cognitive reserve, neural reserve, proxy measure, Alzheimer's disease, neuroimaging

Received: March 02, 2017; Accepted: March 24, 2017; Published: March 27, 2017 


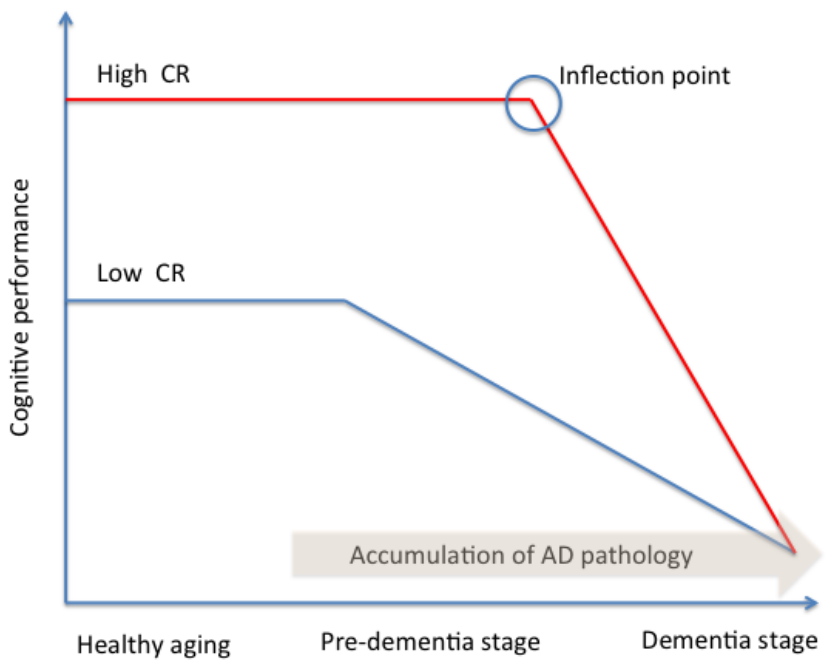

Figure 1. This figure illustrates the different clinical $\mathrm{AD}$ onset according with low and high CR levels. In particular, in patients with high CR the onset of dementia is postponed compared to those with low CR [3]. See text for further details.

Abbreviations: $\mathrm{AD}=$ Alzheimer's disease; $\mathrm{CR}=$ cognitive reserve

activity is modelled by cognitive, social and physical activities experienced during life. This conceptualization introduced a new way to concept the reserve's mechanisms, based on the integrity of functional brain networks, rather than on the brain structure. Therefore, more recently, together with the $\mathrm{CR}$, the "neural reserve" concept has been proposed [5]. Neural reserve involves cognitive networks' efficiency and it provides the neural basis of CR. Individual differences in networks' efficiency, or the use of alternative strategies, may provide reserve against the impact of brain damage. The introduction of the neural reserve concept reduces the distance between BR and CR hypotheses, and it makes differences more nuanced [5].

\section{Static and dynamic reserve's indexes}

Reserves can be built during life through enrichment experiences [5]. These experiences regard all aspects of the individual life. Stern hypothesised that environmental factors, such as years of formal education, occupational attainment and leisure activities, may be considered as proxy measures of CR [5]. Also cognitive performances, such as the intelligence quotient (IQ) [12], memory performances, executive functioning, have been considered as proxy measures of CR [5]. More recently, reserve indexes have been categorised as static and dynamic proxy measure [13-16]. Years of formal education, occupational attainment, pre-morbid IQ and leisure activities pursued during childhood-adolescence and early adulthood life period, have been considered static measures, because they are not directly able to capture the individual cognitive changes. Conversely, memory performances and executive functioning have been considered as dynamic measure because they reflect directly cognitive measure [13-16].

However, among all static CR measures, education is considered the most relevant factor impacting on brain resilience [5,17]. Indeed, it has been stated that education may influence the development of $\mathrm{AD}$ in several ways. Education may induce an increase of synaptic density in the neocortex, causing a delay in the AD onset [18]. Obviously, the deterioration occurs independently of education, but the educational level may reflect cognitive capacity that allow to delay the clinical symptoms of $\mathrm{AD}$, which does not become evident until the deterioration reaches a more severe degree. As a consequence, more $\mathrm{AD}$ pathology is needed to cause dementia in patients with higher educational level [5] (Figure 1). In addition, education could also be considered as a socializing process, promoting learning strategies, encouraging the development of divergent thinking and enabling the individual to perform more competently on cognitive demand [19]. Moreover, education appears to be an important environmental experience that may enhance neural connectivity as well as the propensity to engage in mentally stimulating activities throughout life [20]. Finally, education may improve the intellectual approach to life events [21], which can lead to lifelong mental stimulation and an enhanced activation of the brain regions involved in cognitive processing [21].

Several studies showed a decreased risk of developing $\mathrm{AD}$ in individuals employed in highly mentally demanding occupation $[11,22,23]$. Conversely, individuals without a lifetime occupation showed an increased risk of AD [24]. Evans and co-workers [22] showed that subjects with lower socioeconomic status, computed considering education, occupational attainment and income, had a higher risk of developing AD. Also Stern et al. [11] suggested that the risk of developing $\mathrm{AD}$ was stronger in subjects with both low education and low lifetime attainment, supporting a synergic effect of education and occupation.

Previous studies reported that high level of leisure activities performed during life whether cognitive, social and also physical; reduce the risk of developing dementia [5,25-27]. The following leisure activities are considered "reserve-builders":

1) Cognitive activities, such as reading books, reading magazines and newspapers, producing art (e.g., painting, poetry, sculpture, song writing), produce non-artistic writing (e.g., diary, newsletter, etc.), attending lectures or organized discussions [5,25,27,28].

2) Social activities, such as playing structured games (e.g., cards, board games, crossword puzzles, etc.), participating in hobbies (e.g., gardening, model building, sewing, etc.), volunteering, entertaining friends or relatives $[5,25,27,28]$.

3) Physical activities, such as running, trekking, swimming, biking, skiing, dancing, yoga, etc $[5,25,27,28]$. In addition, healthy life styles, such as Mediterranean diet, responsible drinking, limiting caffeine, nicotine and avoids big-meals, sleep hygiene, etc., are considered important reserve-enhancing factors. Several articles showed that patients with $\mathrm{AD}$ usually had few hobbies and were less involved in psychosocial activities during their lifetime [29-32]. Individuals engaged in frequent cognitive activities such as reading, watching television or card playing were less likely to develop $\mathrm{AD}$ than subjects with infrequent activities [30]. More recently, an increased risk to develop AD in amnesic MCI (a-MCI) patients with low CR level was shown, by using a comprehensive questionnaire investigating cognitive, social, physical leisure activities, education and occupation [27]. In addition, this study revealed that a-MCI patients with high CR and high baseline general cognitive efficiency (as measured by MMSE), showed a significant difference in the survival's time compared to patients with high CR but low baseline MMSE. Specifically, patients with high CR and high baseline MMSE remained AD-free for 21 months respect to those high CR and low baseline MMSE. Conversely, no difference in the survival's time was found in a-MCI patients with low CR and different level of baseline cognitive efficiency. Moreover, a longitudinal study in a large cohort of elderly showed that enriched cognitive life styles both reduced the risk of cognitive decline and improved the recovery of cognitive functions in the early stage of neurodegenerative diseases [33]. An association between complexity of occupation and successful 
aging was also found, supporting the hypothesis that more stimulating environments preserve cognitive ability later in life [34]. However, the combination of different measures of CR (such as education, occupation and cognitive lifestyle) was found more sensitive to reduce the $\mathrm{AD}$ risk, with respect to the different CR proxies separately [25].

However, the above-mentioned static measures have been recently considered as imprecise and few sensitive. Indeed, all these measures are not able to assess specifically the changes in patient's cognition, and they may relate to cognitive performance for reasons other than the reserve mechanisms [13]. For instance the same level of education or of occupational attainment does not reflect the same experience in all individuals [13]. Reed and co-workers [13] proposed the concept of dynamic CR, measured in terms of changes in memory functions. Zahodne and colleagues [14,15] and Serra and co-workers [16] extended the concept of dynamic CR. These studies quantified CR as residual variance of memory scores, after accounting for demographical and brain damage variables. The residual method is in line with a definition of CR [13] as discrepancy between observed performance and expected level of performance. Therefore, individuals who perform better than predicted show positive residual score. It means that they have high CR. Conversely, subjects who perform worse than predicted show negative residual score and they have low $\mathrm{CR}$. The residual variable (expressing the CR) differs from the observed score (in this case quantified as memory performance) because any variance related to demographics, brain damage and cognitive efficiency was ruled out, in addition to error. To conceptualize CR in terms of residual variance of memory functions made the CR's concept more flexible and adaptable to cognitive changes. However, Serra and co-workers [16] have recently shown that indices of dynamic CR that strip off any contribution of general cognitive abilities to retain exclusively memory processes are not able to identify the conversion to $\mathrm{AD}$ efficiently. The integrity of memory function is not sufficient to withstand neurodegeneration [16]. By contrast, the ability to use synergistically the different cognitive functions is protective against the conversion to $\mathrm{AD}$. Using $\mathrm{CR}$ measures limited to assess only memory function is likely less sensitive to detect the cognitive decline and to predict patients' conversion [16]. According with this hypothesis dynamic CR needs a measure of general cognition to identify $\mathrm{AD}$ conversion efficiently. However, a dynamic concept of CR fits better than the more "static" measures based on education or lifestyle indicators with the cognitive changes due to aging and neurodegeneration.

\section{The interaction between reserves and $\mathrm{AD}$-related biomarkers}

Reserve's mechanisms cannot act independently from others biomarkers to cope the effect of $\mathrm{AD}$ neuropathology. Previous studies showed several significant interaction effects between levels of CR, CSF-biomarkers ( $\beta$-amyloid and tau levels), genetic AD-like phenotype (APOE status), brain atrophy and the risk to develop the clinical symptoms of AD [27,35-38]. In particular, Jack and co-workers [35] showed that people with a high risk of cognitive impairment due to $\mathrm{AD}$ pathophysiological processes, have both $\mathrm{APOE}_{4}$ and low $\mathrm{CR}$ levels. On the contrary, individuals with low genetic AD risk and high CR levels better withstand AD pathology and, therefore, maintain longer normal cognitive functions. An interaction between $\mathrm{CR}$, tau and phosphorylated tau (p-tau), but not $\beta$-amyloid levels, was demonstrated [37]. However, also an additive, rather than interacting, effect was reported for CR levels and AD biomarkers [39].

CSF biomarkers are increasingly used in clinical settings, but they requires invasive procedures. In parallel, quantitative neuroimaging is gaining an increasingly central role in the management and the understanding of neurodegenerative dementia. In clinical settings, Positron Emission Tomography (PET) imaging allows to detect patterns of hypometabolism suggestive for specific forms of neurodegenerative dementia [40], while ligands to specific neurobiological substrates, such as the deposition of beta-amyloid plaques [41] or intra-neuronal accumulation of tau-proteins [42], have further increased the potential of detecting specific pathological brain abnormalities in vivo.

Several neuroimaging studies have provided in vivo evidences that lifestyle acts on brain organization, confirming both BR and CR concepts. For instance, Garibotto and coworkers [43], using FDG-PET, showed positive correlation between CR measures (as education, and occupation) and cholinergic activity in the bilateral hippocampus and in the right posterior cingulate gyrus, both in $\mathrm{AD}$ and $\mathrm{MCI}$ patients. The Authors affirmed that the significant correlation found between cholinergic activity, in structures traditionally involved in memory, and $\mathrm{CR}$ proxies, suggests that $\mathrm{BR}$ in $\mathrm{AD}$ is associated with a preserved/ stimulated cholinergic neurotransmission [43].

Recently, other studies showed a significant association between CR proxies, $\beta$-amyloid levels measured in CSF, and FDG-PET metabolism, in patients with preclinical $\mathrm{AD}[44,45]$. In $\mathrm{AD}$ patients $\mathrm{A} \beta$-carriers, higher education was associated with an altered metabolism, as measured by FDG-PET, suggesting that CR plays a compensatory function to sustain cognitive ability in presence of early AD pathology $[44,45]$. An association between FDG-PET metabolism in the temporoparietal cortex and high CR levels was observed also in MCI converters compared to MCI non-converters and controls [46]. Similar results were found also in prodromal AD [47].

Magnetic resonance imaging (MRI) is less expensive and noninvasive, making it an attractive alternative to nuclear medicine. In particular, quantitative MRI is able to detect non-invasively a range of tissue parameters, which are believed to reflect various pathophysiological aspects of brain damage, which may play different roles at different disease stages.

Several structural neuroimaging studies provided strong evidence of the relationship between CR levels and brain resilience. In particular, by using voxel-based morphometry (VBM), AD patients with high $\mathrm{CR}$ (measured as years of formal education) showed significant grey matter atrophy in the entorhinal cortex and in the temporal pole, while less educated $\mathrm{AD}$ patients showed widespread atrophy in the supramarginal gyrus, posterior cingulate cortex and precuneus [36]. Moreover, an association between GM volumes and reasoning abilities was found according to the CR levels. Another study [48] showed that education counteracts the effect of hippocampal atrophy, but of cerebrovascular disease as well in patients with AD. Similarly, Brickman and co-workers [49] provided evidence that healthy elderly with high CR suffered from a severe white matter damage compared with those low CR, suggesting that they need to accumulate more neuropathology to show a lower level of cognitive efficiency. More recently, a significant higher risk for $\mathrm{AD}$ conversion was showed in a-MCI patients with higher CR levels (compared to those with low CR level) and white matter lesions in associative fasciculi [27]. Brain structural damage measured in terms of cortical thickness has been correlated to different levels of CR [50,51]. In particular, $\mathrm{MCI}$ patients that could convert to $\mathrm{AD}$ 6-12 months later, showed low CR levels and significant reduced cortical thickness at baseline, as compared to non-converter MCI [50]. More recently, Serra and co-workers [16] showed in patients with a-MCI, a different association between regional GM volumes and different measures of dynamic CR, one including memory and general cognitive efficiency 
scores (LT $\left.{ }^{1 s t}\right)$ and one including memory score only (d-CR). In particular, GM volumes change in the anterior (ACC) / posterior (PCC) cingulate cortex, in the left hippocampus and parahippocampal gyrus was found to be associated with all used dynamic CR measures. The common brain areas observed were likely involved both in the memory and in the more general cognitive efficiency processes [16]. Conversely, GM volumes change in the precuneus associated with the index including memory and general cognitive efficiency (LT ${ }^{1 s t}$ ), confirming the role played by the precuneus in the general cognitive abilities [16], and highlighting the presence of compensative mechanisms that allow the a-MCI patients with high CR to cope better with neurodegenerative process [16]. Moreover, the index expressing only the memory process (d-CR) associated specifically with the middle anterior cingulate cortex (pm-ACC), with the superior frontal gyrus bilaterally, with the right orbitofrontal cortex and with the right superior temporal gyrus. The pm-ACC has been found to relate with a rapid cognitive response not reflecting the correctness of the response but the cognitive activation only. Nevertheless the right orbitofrontal cortex is typically related to the go-no-go tasks and atrophy in this brain area may be lead to the inhibition deficits. The Authors hypothesised that the patients reaching positive values in the $\mathrm{d}-\mathrm{CR}$ index (and consequently high CR) showed more cognitive promptness, but not necessary more accuracy in the response (as typically observed in patients with low CR). More interesting, although The Authors did not perform a statistical formal comparison of the association between GM volume and the dynamic CR scores between a-MCI and healthy elderly (HE), a qualitative analysis revealed that $\mathrm{LT}^{1 \mathrm{st}}$ was mainly associated, in both groups, with the volume of PCC/precuneus and ACC. Conversely the residual score (expressing the $\mathrm{d}$-CR) was mainly associated with the volume of the most anterior portion of ACC (Figure 2). These overlapping results confirm that the integrity of the cingulate cortex is crucial for cognitive efficiency.

Resting-state fMRI (RS-fMRI) allows investigating functional brain networks and assessing functional connectivity between different brain areas. Relationship between CR and brain networks, which are the closest representations we can obtain of neural reserve in vivo, have

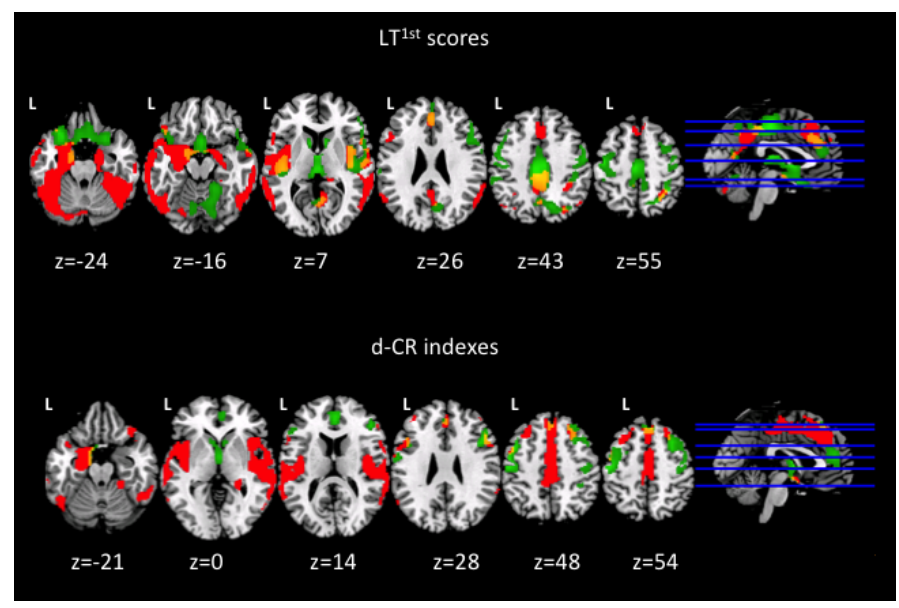

Figure 2. Overlap between regional GM volumes in patients with a-MCI and healthy elderly individuals.

In yellow are shown the overlapping brain areas between a-MCI patients and HE. When considering the $\mathrm{LT}^{1 \mathrm{st}}$ scores we found associations mainly with the $\mathrm{PCC} /$ precuneus and ACC, bilaterally. Moreover, the d-CR indexes we found association with the most anterior part of the ACC and in the left amygdala. Specific brain areas of association were also found in a-MCI patients (in red) and in HE group (in green). See text for further details. Abbreviations: $\mathrm{ACC}=$ anterior cingulate cortex; $\mathrm{a}-\mathrm{MCI}=$ amnestic mild cognitive impairment; $\mathrm{d}-\mathrm{CR}=$ dynamic cognitive reserve; $\mathrm{HE}=$ healthy elderly; $\mathrm{LT}^{\mathrm{lst}}=$ the first latent variable; $\mathrm{PCC}=$ posterior cingulate cortex. been assessed in several studies. RS-fMRI data can be analysed using different methodological approaches, both by using regions of interest(RoI) and by using whole-brain analyses.

Bozzali and co-workers [38], by using a RoI-based RS-fMRI approach, showed that CR modulated the functional connectivity in the posterior cingulate cortex, whose disconnection with the temporal lobes is known to be critical for the conversion from MCI to AD. This effect was highly significant in $\mathrm{AD}$ patients, less evident in patients with MCI, and totally absent in healthy subjects [38]. The Authors hypothesised that CR effects observed in patients were primarily the result of neural compensation (i.e., compensatory resources recruited to cope with brain change) rather than neural reserve (i.e., pre-existing resources determining individual performance in the absence of pathology) [5,38].

An other promising technique is based on the whole-brain analysis driven by graph theory [52,53], a mathematical approach that describes complex systems as networks [52,53]. In simple words, the brain is conceptualized as a number of regions (nodes) that are functionally connected to each other by edges, and whose importance and efficiency within the whole network is determined by their functional specialization (i.e., segregation) and integration. In this view, some nodes are more critical (i.e., centrality) for information processing (efficiency in information transferring) and are called "hubs". Abnormal connectivity between "hubs" is believed to cause more deficits than that between peripheral nodes [52,53]. Recently, Serra and co-workers [16], showed connectivity differences between high and low CR only in a-MCI patients, by using the graph theory [52,53]. In particular, participants with high CR showing a significant increase of connectivity in a network involving mainly fronto-parietal nodes. Conversely, they showed significantly decreased connectivity in a network involving fronto-temporo-cerebellar nodes. These findings supported the hypothesis that CR impacts on neurodegenerative process in the early phase of $\mathrm{AD}$ only. In addition, results fit with the existence of a "neural reserve", characterised by specific neural networks and their efficiency.

\section{Conclusions}

Literature supports the existence of reserve mechanisms that withstand neuropathology. Brain, cognitive and more recently, neural reserve have been documented using different approaches. Interactions between reserve and several biomarkers have been proved. Therefore, it is becoming clearer that healthy lifestyles impact on brain networks. Cognitive functioning and its efficiency in the presence of $\mathrm{AD}$ pathology reflect the efficiency of these networks. It remains to be demonstrated whether interventions later in life can modulate brain resilience. Several studies assessing the influence of different proxies of $\mathrm{CR}$ on the clinical manifestation of $\mathrm{AD}$ indicate that cognitive function is dynamic, rather than static [20]. This is congruent with the "use or lose it" paradigm [54]. According with Hultsch and co-workers [54] cognitive processes are considered modifiable also by exercise and experience, rather than innate factors. In this view, structural and functional brain changes are driven by the exposition to stimuli promoting learning and reinforcing the exiting neuronal connections [5]. In order to sustain the brain plasticity, and therefore to maintain the $\mathrm{CR}$, cognitive rehabilitation in patients with early stage of $\mathrm{AD}$ can be considered a promising therapeutic target. This perspective assumes that a mentally stimulating lifestyle modifies the routes used by the brain to perform successfully a cognitive function. As a consequence, an intensive mental training might provide a greater resilience to cope 
with neuronal damage, likely providing alternative cognitive strategies or recruiting different brain networks [5].

Currently there is a rich literature on cognitive rehabilitation in $\mathrm{AD}$ patients [55], but there are no several studies that explore directly the effect of CR on cognitive outcome after rehabilitation. Olazarán and co-workers [56] only, investigating both $\mathrm{AD}$ and $\mathrm{MCI}$ patients, found better cognitive outcomes after cognitive stimulations in patients with low, compared with those with high educational level. This surprising result is likely due to the fact that in patients with high CR is needed accumulate more $\mathrm{AD}$ neuropathology to show same clinical symptoms of patients with low CR. The Authors [56] concluded that patients with high $\mathrm{CR}$ were less able to learn new generalizable strategies useful to improve cognitive performances. However, further studies assessing relationship between different CR levels and non-pharmacological treatments seem to be necessary and desirable in the immediate future.

\section{Acknowledgment}

This project has been supported by grants from the Italian Ministry of Health to Dr. Marco Bozzali (150/RF-2009-1491699; 047/RF-2010).

\section{References}

1. Nelson PT, Braak H, Markesbery WR (2009) Neuropathology and cognitive impairment in Alzheimer disease: a complex but coherent relationship. J Neuropathol Exp Neurol 68: 1-14. [Crossref]

2. Stern Y, Alexander GE, Prohovnik I, Mayeux R (1992) Inverse relationship between education and parietotemporal perfusion deficit in Alzheimer's disease. Ann Neurol 32: 371-375. [Crossref]

3. Stern Y (2009) Cognitive reserve. Neuropsychologia 47: 2015-2028. [Crossref]

4. [No authors listed] (1940) Reserve Capacity of the Brain. Br Med J 2: 673-674. [Crossref]

5. Stern Y (2012) Cognitive reserve in ageing and Alzheimer's disease. Lancet Neurol 11: 1006-1012. [Crossref]

6. Katzman R, Terry R, DeTeresa R, Brown T, Davies P, et al. (1988) Clinical, pathological, and neurochemical changes in dementia: a subgroup with preserved mental status and numerous neocortical plaques. Ann Neurol 23: 138-144. [Crossref]

7. Schofield PW, Logroscino G, Andrews HF, Albert S, Stern Y (1997) An association between head circumference and Alzheimer's disease in a population-based study of aging and dementia. Neurology 49: 30-37. [Crossref]

8. Mortimer JA, Borenstein AR, Gosche KM, Snowdon DA (2005) Very early detection of Alzheimer neuropathology and the role of brain reserve in modifying its clinical expression. J Geriatr Psychiatry Neurol 18: 218-223. [Crossref]

9. Stern Y (2002) What is cognitive reserve? Theory and research application of the reserve concept. J Int Neuropsychol Soc 8: 448-460. [Crossref]

10. Barulli D, Stern Y (2013) Efficiency, capacity, compensation, maintenance, plasticity: emerging concepts in cognitive reserve. Trends Cogn Sci 17: 502-509. [Crossref]

11. Stern Y, Gurland B, Tatemichi TK, Tang MX, Wilder D, et al. (1994) Influence of education and occupation on the incidence of Alzheimer's disease. JAMA 271: 10041010. [Crossref]

12. Rentz DM, Locascio JJ, Becker JA, Moran EK, Eng E, et al. (2010) Cognition, reserve, and amyloid deposition in normal aging. Ann Neurol 67: 353-364. [Crossref]

13. Reed BR, Mungas D, Farias ST, Harvey D, Beckett L, et al. (2010) Measuring cognitive reserve based on the decomposition of episodic memory variance. Brain 133: 21962209. [Crossref]

14. Zahodne LB, Manly JJ, Brickman AM, Siedlecki KL, Decarli C, et al. (2013) Quantifying cognitive reserve in older adults by decomposing episodic memory variance: replication and extension. J Int Neuropsychol Soc 19: 854-862. [Crossref]

15. Zahodne LB, Manly JJ, Brickman AM, Narkhede A, Griffith EY, et al. (2015) Is residual memory variance a valid method for quantifying cognitive reserve? A longitudinal application. Neuropsychologia 77: 260-266. [Crossref]

16. Serra L, Bruschini M, Di Domenico C, Bechi Gabrielli G, Marra C, et al. (2017) Memory is not enough: the neurobiological basis od dynamic cognitive reserve. Journal of Alzheimer Disease.
17. Dufouil C, Alpérovitch A, Tzourio C (2003) Influence of education on the relationship between white matter lesions and cognition. Neurology 60: 831-836. [Crossref]

18. Katzman R (1993) Education and the prevalence of dementia and Alzheimer's disease. Neurology 43: 13-20. [Crossref]

19. Gilleard CJ (1997) Edication and Alzheimer's disease: a review of recent internationa epidemiological studies. Aging Ment Health 33-46. [Crossref]

20. Vance DE, Crowe M (2006) A proposed model of neuroplasticity and cognitive reserve in older adults. Activ Adapt Aging 30: 61-79. [Crossref]

21. Friedland RP (1993) Epidemiology, education, and the ecology of Alzheimer's disease. Neurology 43: 246-249. [Crossref]

22. Evans DA, Hebert LE, Beckett LA, Scherr PA, Albert MS, et al. (1997) Education and other measures of socioeconomic status and risk of incident Alzheimer disease in a defined population of older persons. Arch Neurol 54: 1399-1405. [Crossref]

23. Qiu C, Karp A, von Strauss E, Winblad B, Fratiglioni L, et al. (2003) Lifetime principal occupation and risk of Alzheimer's disease in the Kungsholmen project. Am J Ind Med 43: 204-211. [Crossref]

24. Anttila T, Helkala EL, Kivipelto M, Hallikainen M, Alhainen K, et al. (2002) Midlife income, occupation, APOE status, and dementia: a population-based study. Neurology 59: 887-893. [Crossref]

25. Valenzuela M, Brayne C, Sachdev P, Wilcock G, Matthews F (2011) Cognitive lifestyle and long-term risk of dementia and survival after diagnosis in a multicenter populationbased cohort. Am J Epidemiol 173: 1004-1012. [Crossref]

26. Sattler C, Toro P, Schönknecht P, Schröder J (2012) Cognitive activity, education and socioeconomic status as preventive factors for mild cognitive impairment and Alzheimer's disease. Psychiatry Res 196: 90-95. [Crossref]

27. Serra L, Musicco M, Cercignani M, Torso M, Spanò B, et al. (2015) Cognitive reserve and the risk for Alzheimer's disease: a longitudinal study. Neurobiol Aging 36: 592600. [Crossref]

28. Sumowski JF, Leavitt VM (2013) Cognitive reserve in multiple sclerosis. Mult Scler 19: 1122-1127. [Crossref]

29. Friedland RP, Fritsch T, Smyth KA, Koss E, Lerner AJ, et al. (2001) Patients with Alzheimer's disease have reduced activities in midlife compared with healthy controlgroup members. Proc Natl Acad Sci U S A 98: 3440-3445. [Crossref]

30. Wilson RS, Bennett DA, Bienias JL, Aggarwal NT, Mendes De Leon CF, et al. (2002) Cognitive activity and incident AD in a population-based sample of older persons. Neurology 59: 1910-1914. [Crossref]

31. Crowe M, Andel R, Pedersen NL, Johansson B, Gatz M (2003) Does participation in leisure activities lead to reduced risk of Alzheimer's disease? A prospective study of Swedish twins. J Gerontol B Psychol Sci Soc Sci 58: P249-255. [Crossref]

32. Verghese J, Lipton RB, Katz MJ, Hall CB, Derby CA, et al. (2003) Leisure activities and the risk of dementia in the elderly. $N$ Engl J Med 348: 2508-2516. [Crossref]

33. Marioni RE, van den Hout A, Valenzuela MJ, Brayne C, Matthews FE (2012) Active cognitive lifestyle associates with cognitive recovery and a reduced risk of cognitive decline. J Alzheimers Dis 28: 223-230. [Crossref]

34. Smart EL, Gow AJ, Deary IJ (2014) Occupational complexity and lifetime cognitive abilities. Neurology 83: 2285-2291. [Crossref]

35. Jack CR Jr, Knopman DS, Jagust WJ, Petersen RC, Weiner MW, et al. (2013) Tracking pathophysiological processes in Alzheimer's disease: an updated hypothetical model of dynamic biomarkers. Lancet Neurol 12: 207-216. [Crossref]

36. Serra L, Cercignani M, Petrosini L, Basile B, Perri R, et al. (2011) Neuroanatomica correlates of cognitive reserve in Alzheimer disease. Rejuvenation Res 14: 143-151. [Crossref]

37. Soldan A, Pettigrew C, Li S, Wang MC, Moghekar A, et al. (2013) Relationship of cognitive reserve and cerebrospinal fluid biomarkers to the emergence of clinical symptoms in preclinical Alzheimer's disease. Neurobiol Aging 34: 2827-2834. [Crossref]

38. Bozzali M, Dowling C, Serra L, Spanò B, Torso M, et al. (2015) The impact of cognitive reserve on brain functional connectivity in Alzheimer's disease. J Alzheimers Dis 44: 243-250. [Crossref]

39. Vemuri P, Weigand SD, Przybelski SA, Knopman DS, Smith GE, et al (2011) Cognitive reserve and Alzheimer's disease biomarkers are independent determinants of cognition. Brain 134: 1479-1492. [Crossref] 
40. Kato T, Inui Y, Nakamura A, Ito K (2016) Brain fluorodeoxyglucose (FDG) PET in dementia. Ageing Res Rev 30: 73-84. [Crossref]

41. Villemagne VL (2016) Amyloid imaging: Past, present and future perspectives. Ageing Res Rev 30: 95-106. [Crossref]

42. Choe YS, Lee KH (2015) PET Radioligands for Imaging of Tau Pathology: Current Status. Nucl Med Mol Imaging 49: 251-257. [Crossref]

43. Garibotto V, Tettamanti M, Marcone A, Florea I, Panzacchi A, et al. (2013) Cholinergic activity correlates with reserve proxies in Alzheimer's disease. Neurobiol Aging 34: 2694. [Crossref]

44. Garibotto V, Borroni B, Sorbi S, Cappa SF, Padovani A, et al. (2012) Education and occupation provide reserve in both ApoE ?4 carrier and noncarrier patients with probable Alzheimer's disease. Neurol Sci 33: 1037-1042. [Crossref]

45. Ewers M, Insel PS, Stern Y, Weiner MW (2013) Cognitive reserve associated with FDG-PET in preclinical Alzheimer disease. Neurology 80: 1194-1201. [Crossref]

46. Garibotto V, Borroni B, Kalbe E, Herholz K, Salmon E, et al. (2008) Education and occupation as proxies for reserve in aMCI converters and AD: FDG-PET evidence. Neurology 71: 1342-1349. [Crossref]

47. Morbelli S, Perneczky R, Drzezga A, Frisoni GB, Caroli A, et al. (2013) Metabolic networks underlying cognitive reserve in prodromal Alzheimer disease: a European Alzheimer disease consortium project. J Nucl Med 54: 894-902. [Crossref]

48. Murray AD, Staff RT, McNeil CJ, Salarirad S, Ahearn TS, et al. (2011) The balance between cognitive reserve and brain imaging biomarkers of cerebrovascular and Alzheimer's diseases. Brain 134: 3687-3696. [Crossref]
49. Brickman AM, Siedlecki KL, Muraskin J, Manly JJ, Luchsinger JA, et al. (2011) White matter hyperintensities and cognition: testing the reserve hypothesis. Neurobiol Aging 32: 1588-1598. [Crossref]

50. Querbes O, Aubry F, Pariente J, Lotterie JA, Démonet JF, et al. (2009) Early diagnosis of Alzheimer's disease using cortical thickness: impact of cognitive reserve. Brain 132: 2036-2047. [Crossref]

51. Arenaza-Urquijo EM, Molinuevo JL, Sala-Llonch R, Solé-Padullés C, Balasa M, et al. (2013) Cognitive reserve proxies relate to gray matter loss in cognitively healthy elderly with abnormal cerebrospinal fluid amyloid-? levels. J Alzheimers Dis 35: 715726. [Crossref]

52. Bullmore E, Sporns O (2009) Complex brain networks: graph theoretical analysis of structural and functional systems. Nat Rev Neurosci 10: 186-198. [Crossref]

53. Rubinov M, Sporns O (2010) Complex network measures of brain connectivity: uses and interpretations. Neuroimage 52: 1059-1069. [Crossref]

54. Hultsch DF, Hertzog C, Small BJ, Dixon RA (1999) Use it or lose it: engaged lifestyle as a buffer of cognitive decline in aging? Psychol Aging 14: 245-263. [Crossref]

55. Sitzer DI, Twamley EW, Jeste DV (2006) Cognitive training in Alzheimer's disease: a meta-analysis of the literature. Acta Psychiatr Scand 114: 75-90. [Crossref]

56. Olazarán J, Muñiz R, Reisberg B, Peña-Casanova J, del Ser T, et al. (2004) Benefits of cognitive-motor intervention in MCI and mild to moderate Alzheimer disease. Neurology 63: 2348-2353. [Crossref]

Copyright: $\odot 2017$ Serra L. This is an open-access article distributed under the terms of the Creative Commons Attribution License, which permits unrestricted use, distribution, and reproduction in any medium, provided the original author and source are credited. 